\title{
Reflections on Computer-Mediated Architectural Design
}

\author{
Thomas Kvan
}

\begin{abstract}
The application of computer tools to mediating and promoting collaborative design efforts between mutually distant parties has become feasible. Technology is again ahead of practice, and problems of assimilation have only begun to be explored. This paper postulates the requirements of environments for computer-mediated collaborative design in architectural practice, drawing upon experiences of design collaboration among schools of architecture on three continents and supplementing these with enquiries into design excellence in practice.
\end{abstract}

\section{Some Tales of ARChitectural Design}

A RCHITECTURE is a solitary art, or so the story goes. In this version of events, the architect imagines possible inventions, wrestles with the act of creation, and delivers to the world a design formed of his or her imagination. Fostered by popular fiction such as Ayn Rand's The Fountainhead, this image of the architect has been fashionable of late in Hollywood, with a number of movies exploiting the theme of an isolated genius and the image of the lone designer at an easel, attended by the muse.

At the other extreme, architects in both design practices and corporate or governmental offices are portrayed as members of teams in which individual contribution is barely discernible at the conclusion of particular projects. Projects take years to complete, and the compositions of teams change as transitory members contribute their specialist knowledge at appropriate moments before moving on. This version of practice is caricatured typically by a large drafting room filled with workers with eyeshades and rolled-up sleeves bent over drafting boards (or the modern equivalent, bending over computer-aided design-CAD—workstations).

Although both images are faithful illustrations of some aspect of professional practice (but certainly cannot be taken literally), neither tells the whole story. They both fail to portray the true social nature of the profession toward the end of the twentieth century [1].

The network within which architects work has expanded throughout the history of the profession, extending out from community to region and country; now, almost any architectural commission is executed within a global setting, in particular a global financial context. Participants in the process include colleagues, peers, consultants, a vast web of funding sources, and the multitude of regulatory or supervisory agents. Architecture is, and always will be, practiced in a framework that extends beyond the walls of the studio or office. Indeed,

Manuscript received June 1994; revised August 1994

The author is with the Department of Architecture, University of Hong Kong, Hong Kong.

IEEE Log Number 9407672. architecture has always been practiced in a format that has recently come to be known as a virtual office, that is, a constantly changing composition of participants, joining together to realize a project or phase of a project, and reconfiguring participants for the next piece of work. Typically, this consists of colleagues and consultants located near each other, though most major projects include consultants from out of town or out of state. Teams use a variety of techniques to overcome the obstacles that arise from disparate locations, unfamiliarity with team members, poor group dynamics, and other hindrances to successful collaboration.

The capacity of electronic tools to facilitate this method of working and transport a designer to a remote project have progressed dramatically, yet the benefits derived from these systems have not advanced beyond early visions, such as this one by Wiener:

Let us suppose we have an architect in Europe supervising the construction of a building in the United States. . . Let him draw up his plans and specification as usual. Ultrafax gives a means by which a facsimile of all the documents may be transmitted in a fraction of a second, and the received copies are quite as good as working plans as the originals. The architect may be kept up to date with the progress of the work by photographic records taken every day or several times a day, and these may be transmitted back to him by Ultrafax. Any remarks or advice he cares to give his representative may be transmitted by telephone, Ultrafax, or teletypewriter. [2]

Reading this, you are probably wondering if this is a current advertising campaign for a multimedia joint venture of a Baby Bell. The language sounds quaintly archaic (after all, when was the last time you used a "teletypewriter"?). You probably also wonder what the fuss is about--architects do all these things as a matter of course, even if the project is not in another country but just around the corner.

Although it was written in 1954, Wiener's fantasy is not far from the current practice. One architectural practice with which I have consulted consists of a sole practitioner in a room in his house in Pennsylvania, serving a corporate client in Connecticut by designing and supervising the construction of buildings in Honduras and the Philippines. The practice relies on a laptop computer and a fax machine; all this activity takes place throughout the day as the different time zones come in to operation. This method of practice is not unusual. Many small or sole practices regularly serve clients on substantial projects, teaming as required for a particular task. With telecommunications, the next time zone is as convenient to reach as next door. 
Computer tools are now a norm in almost all architectural offices around the world and the majority of design schools. Surveys carried out by organizations such as the American Institute of Architects suggest that over $90 \%$ of architectural practices in the U.S. now use CAD in some form. My observations find a similar saturation in Asia and Europe. While most implementations are mired in the mundane details of computer-aided drafting, automating only the drawing process and only in cellular, isolated fashion without even local area networks, a few offices have begun to share data over distances and promote collaboration between remote offices. Typically, this collaboration is occurring between branch offices of the same organization or between a few engineering consultants and the architect. The pattern is fairly consistent: one office ships drawings off to another at the end of a discrete phase, such as the architects providing background drawings to structural engineers when the schematic design is completed. Work is done at the recipient's office using the original files as backgrounds; completed work is then plotted to be collated into issued drawing sets. More recently, we have begun to see collaboration between architects in remote offices during the design effort, achieving this, for example, by passing a database of drawings at the end of each working day to another time zone where the day is just beginning and then back again. Thus, a building in Jakarta is currently being designed in Los Angeles with assistance from architects in Singapore knowledgeable of particular Indonesian conditions.

A characteristic of almost all collaboration today is its asynchronous nature. None of the professional implementations to date apply computer tools to interactive synchronous design. Unlike that followed by the team designing a building for Jakarta, a more traditional process would see team members gathering together in one location, dividing up work but staying within speaking distance so that they could coordinate the process. Periodic meetings would be held to review design solutions, with team members leaning over the table and marking up a set of drawings. Major design reviews would see the drawings all pinned up on the wall and marker pens brandished to change, add, or delete. Since collective synchronous design is an essential activity of an architect, it is interesting to observe and speculate how electronic interaction can affect or contribute to design itself. This conjecturing can also be tempered by asking how we might use this technology differently and use it to change practice for the better.

\section{COMPUTER-MEDIATED DESIGN}

There is extensive literature around the question, "What is architectural design?" [3], but there have been few satisfactory descriptions. In all models of the design process, though, we find in common the activities of cogitation, expression/modeling, and communication/testing. Without engaging in discussion about computer-mediated cogitation or speculating on the results of computer-mediated design, we can observe electronic interaction affecting the process of design. In particular, we can consider ways in which computermediated design collaborations might be conducted.

In practice, an architect draws upon a wide variety of media to express his or her ideas. Within the course of a project, an architect can expect to produce drawings using transparent and opaque paper (even the back of an envelope) using pencil, ink, or paint. Models might be created, employing card, clay, plastic, metal, and almost any other material (for example, trees on a model might be made from branches of bushes, sponge, paper, or plastic). A project might be explained using words, numbers, or charts. Contracts and legal documents accumulate, and records are kept of all manner of exchanges of information.

As these data gather, a designer begins to identify constraints, opportunities, references, and allusions that might be important to setting a direction for a solution. Diagrams are drawn for such things as major circulation flows, dominant axes, or structural problems. These will be supplemented by more detailed partial solutions, ranging from how an entrance might work to building components. References may be made to earlier solutions, and searches are made to find these precedents. As this work proceeds, an architect accumulates sketches of space in two or three dimensions, using conventional representations like perspectives, plans, elevations, and sections. Some particularly complex spaces must be created in model form so they can be picked up and turned around, then torn and reglued as ideas evolve. Additional thoughts are represented by studies in texture or light, or by technical data and calculations. Meetings are held with clients, consultants, and colleagues to explore ideas, review progress, and solicit input.

As design ideas are expressed, a significant difference between manual drawings and computer-based images becomes apparent. There is a perceived level of specificity in all computer output, be it word processing or drawings. Laser-printed text looks final, no matter how it is labeled. Aligned pixels seem more definite than smudged graphite. This has some implications for the quality of communication achieved-a vague image invites participation while a crisp line defies involvement. These distinctions can be used to advantage, too-the degree of specificity must change as the design progresses and decisions are made.

At earlier design stages in particular, ambiguity plays a major role, both in communicating the design to others and to oneself. Much of the perceived freedom in traditional materials stems from ambiguity or the ability of each perceiver to interpret results subjectively. It is true that some early design information is precise, that there is little opportunity for reinterpretation on the part of the receiver. Much is vague, however, and reinterpretation is essential to a design dialogue. Lines are tentatively drawn, with the right reserved to change later.

Fortuitous misinterpretation also plays a part in creativity, reminding us that clarity is not desired at all times. Designers can misinterpret their own sketches or annotations as well as communications from their colleagues or even the client. The role of misinterpreted communications in history, science, or politics is perhaps better documented than that in design, but undoubtedly it occurs as frequently. In design communications, thick, broken, or incomplete lines contribute to the ambiguity. This is why many designers prefer to work with soft pencils, rapid marker strokes, and rougher paper first, moving to ink on smooth mylar later. To reintroduce some of the ambiguity into overly precise CAD drawings, some 
users employ software to render the precise digital drawing in one of three line styles - back of the envelope squiggly, freehand-drawn slightly more precise, and hard-line finality!

In digital terms, this suggests, for example, that bit-mapped images appear to hold greater promise at the early stages of a design cycle, whereas the precision of a structured computer graphics model reflects the less flexible phases of a later design stage. All this is compounded by strictures placed by particular software systems, which require the user to act in a particular pattern or sequence. The freedom and spontaneity of traditional media is perceived to be lost in the digital realm both from the means of representation and from the methods of interaction.

\section{COMMUNICATION FOR COLlaborative DESIGN}

Participation in a design process comes in different forms. Some design communication is serial-solutions are posed and countered. In this format, an asynchronous sending back and forth of design files is a perfectly adequate tool. The designers are able to annotate, edit, and supplement the design document(s) as they are traded. At other stages of design, synchronous interaction between participants becomes important, as described earlier. In addition to synchronous and asynchronous communications, there is a model for a third level of exchange. Hollan and Stornetta posit that "semisynchronous mechanisms will encourage a greater range of responses than the normal asynchronous or synchronous mechanisms" [4]. In their model for e-mail exchanges, responses to a publicly posted message would be batched up and released simultaneously at some future time, permitting more original responses and reducing the opportunity for an early response to control the direction of the discussion.

We have a similar tradition in the design world. Teams divide up and tackle the same design problem in smaller groups, looking for a variety of solutions to a problem. After a stipulated time, teams gather again and pin up their sketches for comparative reviews. Thus, a communication is made and multiple replies prepared and dispatched, but none are revealed until all are received. The comparative review is the most important step in this process and one in which all participants must be able to engage openly. Experience would suggest that such semisynchronous communication would reduce the richness of a design interaction if it did not include a very open collective review. Design is not a linear dialogue but proceeds more in the vein of a Joycean communication, leaping to allusions, returning to earlier forms, and worrying over details. Constrained by any fixed process, design is rendered sterile.

We postulate that video plays less of a role than imagined in this form of communication. Video connections in which you see a person's face may help to establish an initial contact, but this can also be achieved by photographs or packaged video statements rather than live video. Compare two setups for design. In one, described by Harriman and Minneman [5] as a media space, collaborating designers have video cameras and displays that show the faces of participants, as well as others that show the work surface. Participants can therefore track facial movements as well as gesticulations or activities on the drawing board. In another setup, we used the Collage software, which offers an interactive whiteboard, complemented with an audio connection. Seeing a hand move over an image during a design modification is less important than seeing the result of the movement (i.e., the line traced). Adding a voice overlay and supplementing this with still video or scanned images provides adequate spontaneity for successful communication. Although it is true that communication in some cultures relies heavily upon gesticulation, effective communication can be achieved without these ancillary references. Our experience suggests that the non-live video configuration is effective in bringing someone into a design process without the high bandwidth required for multiple video connections. This hypothesis appears to be supported by the telecommunications market-for example, AT\&T has recently supplemented its videophone offerings with the Picasso still video system, reflecting a market void not filled by synchronous video communications systems.

A recent exercise helps to shed some light on the role of computers in collaborative design. Six schools of architecture in five different time zones on three continents brought together a group of design students to tackle a common problem. Schools of architecture in Barcelona, British Columbia, and Hong Kong, and at Cornell, Harvard, MIT, and Washington in St. Louis, joined together for two weeks to devise new housing models for an area of Shanghai scheduled for redevelopment. The exercise, known as the Virtual Design Studio (VDS) [6], thus introduced time zones, culture, and geography as variables with which the students had to deal (the term virtual design studio was first used by Dean William J. Mitchell of MIT in a talk at the Media Lab in February 1993).

In this exercise, the students used as tools for communication and collaboration a variety of systems, reflecting the concerns and direction of the individual schools. Each school handled the design task as it wished-some formed teams while others allowed the students to tackle the problems individually. A server was set up in Vancouver to which files for sharing were posted - in effect a digital "pinup board," as it has come to be known [7]. Scanned images, CAD models, and ASCII files were posted to be downloaded using ftp when convenient. Access to the pinup was unrestricted for participants-anything could be uploaded or downloaded as needed. This interaction was supplemented by e-mail messages, which grew to have distribution lists that filled a screen. Further interaction was achieved at different times during the project by using Collage, vat, and CU-SeeMe. (These are shareware systems - Collage v1.2.1 from ftp.ncsa.uius.edu and LBL Visual Audio Tool v2.17beta from ftp.ee.lbl.gov).

Students were asked initially to exchange ideas for design solutions using any media they chose, to comment on each other's solutions, and to encourage discussion of approaches. The second half of the exercise was then used to develop more detailed models and renderings to describe particular design solutions promoted by each team (or individual). An original intention to generate interaction over the designs failed, for reasons explored below. At the end of the two weeks, we held a video conference call bringing together all the participants for two hours of intercontinental presentation and review.

In the pressure of a design deadline, participants reverted to familiar tools. In the end, participants tackled the design 
problem locally, typically off-line, drawing upon a wide variety of media to explore and express notions. These designs were then repackaged digitally when necessary (by scanning, digital photography, or recreation in a modeling system) to send to the pinup board for open discussion. In particular, we noted that collaboration failed to occur when insufficient opportunity was given for participants to establish a familiarity and dialogue that helped to illuminate local interpretations and assumptions. What we experienced was not what we hoped but what, perhaps, experience should have told us to expect.

\section{SUPPORTING DESIGN EXCELLENCE}

Although we can demonstrate that these tools help us design collaboratively, what makes good design happen? How do the participants interact constructively? While this is almost as vague as subject as "What is design?," we are fortunate to have some useful documentation of processes that have led to good design.

The American Institute of Architects sponsored in 1989 a series of roundtable discussions, workshops, panel discussions, and conferences on the subject of excellence in design [8]. Although there are rightly multiple definitions of excellence [9] and likewise many hypotheses on how excellence is achieved, a broad consensus evolved from this effort about some of the conditions for producing design excellence. In general, excellence is achieved when the designer knows the participants and the problem well, and when this leads to a shared definition of the problem with the other participants. In order to reach such a shared definition, the participants of the roundtables noted that they work both individually and collectively to understand the issues and to explore solutions. Indeed, a group of "signature" firms [10] concluded that excellent design projects were characterized by substantial attention to the processes of exploration and gaining trust of those involved in the project, this period of the project being known as predesign work [11].

Another source sheds useful insights on the role of individuals in the design process. After a close review of three case studies that led to favorable outcomes in terms of good design, Dana Cuff has characterized the process as teamwork with independence in which "a team-like sensibility bonded the central players who struggled together to create the excellent outcome, but these individuals did not necessarily participate equally or collaboratively. Instead, key individuals played key roles; their talent and authority was reported to be essential to the building's success" [12]. The participatory process needs to allow individuals the opportunity to find themselves key roles.

In both of these descriptions of achieving excellent design, the role of participatory communication is paramount. Indeed, the interaction is essential to successful design; the Howard Roark attitude to collaboration in The Fountainhead, that collaboration leads to a dilution of intent, and thus mediocrity, is far from the truth. This poses a serious challenge to computermediated design. How can such mediation be achieved without diluting the participation?

One problem apparent in our experiments is the need for participants to know more about those with whom they are working. Design is a personal activity; knowing something about the others on the team can give you clues on how to interpret their sketches. If you accept Cuff's findings, expressions of personal characteristics become even more important as key individuals assert themselves. It is not clear yet how this fits with research into e-mail communications that suggests that electronic conversations attenuate contextual cues, resulting in a reduction in status differences. In the case of written interactions, Sproull has noted, "The results confirmed that the proportion of talk and influence of higherstatus people decreased when group members communicated by electronic mail" [13]. Although this may apply also to design situations, we have not noticed the corollary: "The increased democracy associated with electronic interactions in our experiments interfered with decision making. We observed that three-person groups took approximately four times as long to reach a decision electronically as they did face-toface . . making it impossible for people to interrupt one another slowed decision making and increased conflict as a few members tried to dominate control ... ." [13]. Instead, our experiences in collaborative design have shown a willingness to discuss and accommodate, though we have not tested these in formal comparative experiments.

An experience during the VDS illustrated the power of personalization in communication. The Barcelona students undertook a very formal, algorithmic approach to design, generating an intimidating matrix of permutations. Students used to less formal approaches to design found it difficult to react to this work. Fortunately, Barcelona's students had established an identity and character for open interaction, for fun, by posting images of candy distributed during a carnival that took place during the time of the VDS, supplemented with quotes taken from various texts. This helped recipients of their more intellectually challenging (and forbidding) messages react openly when these came later. These ephemera created an attitude that video cameras or audio connections could not have done, especially in a multilingual (in our case, Spanish and Cantonese), multicultural experiment.

There is one last thing to think about. Design is an egoladen activity. A participant invests in the created object or image a measure of his or her personal being. A collaborative process requires the design contributor to be willing to step back and permit the other participants to amend the design. For a constructive design collaboration to occur, the participants must come to a tacit understanding that their contributions will be valued. The issues of intersubjectivity and the recognition of the fiduciary roles [14] of the participants, looking after the other's concerns, have not been investigated in the design process.

In computer-mediated design, the difficulties of full participation are acutely obvious. Here, the participants must submit to a consignment, in the terms of Vaitkus, "simply submitting and giving oneself over," to the process and the fellow participants. As Vaitkus has noted, it is difficult enough to submit within a known group, but even more difficult when the colleague is unknown: "The milieu cannot readily accept a new and, thus, anonymous member without forsaking its distinctive, private, familiar character which gains its significance precisely in opposition to such anonymous others" [14]. How 
do you operate in an environment in which such anonymous members are by definition always present? What can be done to reduce the level of anonymity? These questions seem to be central to the design of any collaborative system. Any solution that fails to provide means to build trust cannot succeed, regardless of how fluent its interface is.

\section{CONCLUSION}

We are not looking to tools for computer-mediated design to displace physical collaboration but as a way to enhance remote collaboration. Simulating and automating nonmediated processes is not helpful; we need something new. Thus, we must look beyond our current preconceptions of needs for proximity (real or imitated) for interaction and to develop, in the terms described by Hollan and Stornetta, "tools that go beyond being there" [15]. Perhaps the computer-mediated studio could be an even more interesting place than the real one. We have learned from implementations of CAD and drafting systems that imitation of the manual process in design is not appropriate, that it leads to trivialization of the potentials of a medium. Instead, it is more appropriate to discover how the interaction might be different using a particular technology.

Evidence suggests that good professional work comes from shared mutual respect between participants, professional and client. As Cuff noted of the successful interactions she observed, "the design process is characterized by warm, almost familiar relations among the actors, as well as conflict and, at times, tension" [12, p. 234]. If computer-mediated design is to be successful, the tools will need also to mediate the tension, the warmth. Some have suggested that better simulation of immediacy is the answer, especially by using video cameras. At this time, we think that the role of video is overplayed, that there are other tools that will help us achieve this familiarity through action and participation.

The real challenge, though, seems to be in finding new methods of working. While we can design new tools, can we establish new processes to take advantage of these? Experience with CAD systems suggest that this will probably be more difficult than devising appropriate tools. Computer-mediated design changes the practice of architecture and, as noted by Weld Coxe, "The practice of architecture is really one completely interwoven activity, and is a key to excellence" $[11$, p. 98]. We can have no success if we do not consider the whole.

\section{REFERENCES}

[1] See the work of J. R. Blau, Architects and Firms: A Sociological Perspective on Architectural Practice. Cambridge: MIT Press, 1984 and D. Cuff, Architecture: The Story of Practice. Cambridge: MIT Press, 1991.

[2] N. Wiener, The Human Use of Human Beings: Cybernetics and Society. Boston: Houghton Mifflin, 1954, pp. 97-98.

[3] B. Lawson, How Designers Think. London: Architectural Press, 1980.

[4] J. Hollan and S. Stornetta, "Beyond being there," ACM 0-89791-5135/92/0005-0119, reprinted in R. Baecker, Ed., Readings in Groupware and Computer-Supported Cooperative Work. San Mateo, CA: Morgan Kaufman, 1993.

[5] S. Harrison and S. Minneman, "The media space: A research project into the use of video as a design medium," in Proc. Conf. Participatory Design 1990, reprinted in R. Baecker, Ed., Readings in Groupware and Computer-Supported Cooperative Work. San Mateo, CA: Morgan Kaufman, 1993.

[6] This exercise is described in detail in Nancy Cheng et al., "Place, time and the virtual design studio," in Proc. ACADIA Conf., 1994; the author's role was as one of three faculty members involved. Many of the ideas described in this paper were discussed and rehearsed during this exercise, and the author is indebted to his colleagues for their reflections and criticisms.

[7] The Virtual Design Studio was the fourth in a series of collaborative design exercises. Earlier efforts are documented in $\mathbf{J}$. Wojtowicz, $\mathbf{J}$. Davidson, and W. J. Mitchell, "Design as correspondence," in Proc. ACADIA Conf., 1992; and also in J. Wojtowicz, Ed., Virtual Village Studio. Hong Kong: University of Hong Kong Press, 1994.

[8] The discussions were part of the AIA Design Practice for the '90s program, and the results are documented in T. Vonier, Ed., In Search of Design Excellence. Washington, DC: American Institute of Architects, 1989.

[9] The problems inherent in defining "excellence" in architecture are clearly discussed in R. Shibley, "Architectural excellence: Framing the debate," in In Search of Design Excellence, T. Vonier, Ed. Washington, DC: American Institute of Architects, 1989.

[10] The AIA organized two roundtables, one for "signature" firms (those in which an individual talent leaves a unique mark on a body of excellent work) and another for "star" firms (those firms that have left a consistent body of good work, stemming from stable management and design methodologies). These two approaches cross-checked findings and led to strong consensus on preferred approaches to achieve excellence, some of which are discussed in this paper.

[11] W. Coxe, "Role models of excellence: Learnings from the Research Roundtable," in In Search of Design Excellence, T. Vonier, Ed. Washington, DC: American Institute of Architects, 1989.

[12] D. Cuff, Architecture: The Story of Practice. Cambridge: MIT Press, 1991 , p. 241.

[13] L. Sproull and S. Kiesler, "Computers, networks and work," Scientific American, pp. 116-123, Sept. 1991.

[14] S. Vaitkus, How is Society Possible? Dordrecht: Kluwer, 1991

[15] J. Hollan and S. Stometta, "Beyond being there," ACM 0-89791-5135/92/0005-0119, reprinted in Readings in Groupware and ComputerSupported Cooperative Work, R. Baecker, Ed. San Mateo, CA: Morgan Kaufman, 1993.

Thomas Kvan is a lecturer in the Department of Architecture at the University of Hong Kong as well as a partner in The Coxe Group, a management consulting practice serving the design professions throughout the world. He holds an MA from the University of Cambridge and a Masters in Architecture with a specialization in computer-aided design from The University of California, Los Angeles. He has practiced architecture in Hong Kong, Botswana, and the United States. Since 1981, he has consulted to architects and engineers in the United States, Asia, and Europe on a broad range of management concerns, including the application of computer tools to practice. 